\title{
MIMU/GNSS Tightly-Coupled Covariance Shaping Adaptive Filtering Method for UAV
}

\author{
Bin Liu ${ }^{1, a}$, Rongjun Mu ${ }^{1, b}$, Xin Zhang ${ }^{1, \mathrm{c}}$, Naigang Cui ${ }^{1, \mathrm{~d}}$ \\ ${ }^{1}$ School of Astronautics, Harbin Institute of Technology, Harbin, 150001, China \\ aemail: xiaobin_hit@163.com, ${ }^{\mathrm{b} e m a i l}$ : murjun@163.com, ${ }^{\mathrm{c} e m a i l}$ : hitnicole@126.com, ${ }^{\mathrm{d}} \mathrm{email}:$ \\ cui_naigang@163.com
}

Keywords: unmanned aerial vehicle(UAV); adaptive filtering; tightly-coupled; covariance shaping

\begin{abstract}
A covariance shaping adaptive Kalman filtering method is newly presented to improve the positioning accuracy of MIMU/GNSS tightly-coupled integrated navigation system for UAV. A dual channel parallel filter scheme with the adaptive filter is proposed to improve the real-time performance and make the tightly-coupled integrated navigation system perform better in complex and dynamic environments. The gain of the covariance shaping adaptive filter is self-tuned by changing covariance weighting factor, which is calculated by minimizing the cost function of the Frobenius norm. Hardware-in-the-loop simulation results indicate that the positioning accuracy of the adaptive Kalman filter is increased by $32.6 \%$ against the standard one.
\end{abstract}

\section{Introduction}

MIMU/GNSS Tightly-Coupled integrated navigation system has been widely studied and applied to vehicles and aircrafts[1][2]. In the previous work, both loosely-coupled and tightlycoupled architectures have been investigated[3][4]. When provided clear open sky-access and on vehicles with docile dynamics, both approaches have shown the similar estimation performance. Normally, loosely-coupled architecture with a least square filter has better performance than tightly-coupled architecture in convergence and dynamic. However, when confronted with challenging application environments, such as poor visibility, frequent phase breaks, re-capture (e.g. building block and fast attitude dynamics), and high-multi-path, tightly-coupled formulations inherently have the advantage[5].

Rapidly deployable UAV platforms are attractive for many applications that not only require precise positioning and the flexibility for deployment anywhere on the earth, but also often suffer from aforementioned environmental conditions that are unfavorable for achieving the optimal precise positioning performance[6]. In particular, UAVs typically have fast attitude dynamics(i.e. roll or yaw angle approaching $45^{\circ}$ is common), which lead to frequent phase breaks and recapture, poor satellite geometry and phase out of synchronization(the frequent loss and recapture of GNSS signal caused the inconsistency of the phase-locked loop). Therefore, loosely-coupled architectures can not meet the requirements of accuracy and stability. So in order to improve the adaptability for challenging environments, tightly-coupled architecture based on redundant MIMU with FDI and a modified adaptive filter might be a feasible scheme for UAV precise positioning.

Generally, process and measurement noises are not white Gaussian noise, which can degrade the estimation accuracy and performance of Kalman filter. To improve the estimation quality of an error state vector, the method of estimating process noise covariance is proposed[7]. A Frobenius norm with the residual of measurement is formulated as a cost function[8], which should be minimized by evaluating the norm values. However, this could not guarantee the stability of the system because it had assumed the minimal values of variance were zero. To remedy the defeat, a real-time estimation method of process and measurement noise is proposed[9].

In this paper, a modified MIMU/GNSS tightly-coupled integrated navigation model and a covariance shaping adaptive Kalman filter will be studied. Pseudo-range deviations of low elevation GNSS satellite are added into state space to improve the stability of the filter in challenging environments, in which frequent phase breaks and recapture may occur. Meanwhile, the adaptive 
Kalman filter, which minimizes the cost function of a measurement residuals to estimate the optimal error covariance in real time, is applied to the MIMU/GNSS tightly-coupled integrated navigation data fusion system. By shaping the error covariance of the state vector in real time, the adaptive Kalman filter can optimize the estimation accuracy and thereby improve its stability and robustness. The error covariance consists of two parts. One has the fixed initial values and can be updated like conventional Kalman filters. The other has adaptive gains which can be achieved by minimizing the cost function in the form of Frobenius norm. This adaptive filter shows the optimal estimation performance and stability by compensating the errors which arise from the modeling errors of process and measurement noise. Hardware-in-the-loop simulation system then is built, which mainly consists of four redundant MIMUs and multi-channel GNSS receivers(GPS and GLONASS), and the test results prove its effectiveness.

\section{Inertial Navigation System Mechanization}

The mechanization of inertial navigation system(INS) in the geographic coordinate system can be broken up into three steps: attitude update, velocity update and position update, with the fourth order Runge-Kutta method. To perform the attitude update, the differential equation of quaternions is shown in Eq.(1).

$$
\begin{gathered}
\boldsymbol{Q}\left(q_{0}, q_{1}, q_{2}, q_{3}\right)=q_{0}+q_{1} \mathbf{i}+q_{2} \boldsymbol{j}+q_{3} \boldsymbol{k} \\
{\left[\begin{array}{c}
\dot{q}_{0} \\
\dot{q}_{1} \\
\dot{q}_{2} \\
\dot{q}_{3}
\end{array}\right]=\frac{1}{2}\left[\begin{array}{cccc}
0 & -\omega_{x} & -\omega_{y} & -\omega_{z} \\
\omega_{x} & 0 & \omega_{z} & -\omega_{y} \\
\omega_{y} & -\omega_{z} & 0 & \omega_{x} \\
\omega_{z} & \omega_{y} & -\omega_{x} & 0
\end{array}\right]\left[\begin{array}{l}
q_{0} \\
q_{1} \\
q_{2} \\
q_{3}
\end{array}\right]}
\end{gathered}
$$

The updated velocity is calculated by using Eq.(2) with the fourth order Runge-Kutta integrator.

$$
\left\{\begin{array}{l}
\dot{V}_{N}=f_{N}-\left(2 \omega_{i e} \sin \varphi+\frac{V_{E}}{R_{N}} \tan \varphi\right) V_{E}-\frac{V_{N} V_{U}}{R_{M}} \\
\dot{V}_{U}=f_{U}+\left(2 \omega_{i e} \cos \varphi+\frac{V_{E}}{R_{N}}\right) V_{E}+\frac{V_{N}^{2}}{R_{M}}-g \\
\dot{V}_{E}=f_{E}+\left(2 \omega_{i e} \sin \varphi+\frac{V_{E}}{R_{N}} \tan \varphi\right) V_{N}-\left(2 \omega_{i e} \cos \varphi+\frac{V_{E}}{R_{N}}\right) V_{U}
\end{array} \text { and }\left[\begin{array}{c}
f_{N} \\
f_{U} \\
f_{E}
\end{array}\right]=\boldsymbol{C}_{b}^{n} \boldsymbol{f}^{b}\right.
$$

In the $\mathrm{Eq}(2), V_{N}, V_{U}, V_{E}$ is the north, up and east velocity respectively; $\varphi$ is the latitude; $\omega_{i e}=7.292115 \times 10^{-5} \mathrm{rad} / \mathrm{s}$ is the rotational-angular velocity of the earth; $\boldsymbol{f}^{b}$ is the acceleration vector of measurement; $g, R_{N}, R_{M}$ is respectively the gravitational acceleration, prime circle radius and meridian radius of earth, which in the WGS-84 coordinate system can be calculated according to Eq.(3).

$$
\begin{aligned}
& R_{N}=R_{e}\left(1+e \sin ^{2} \varphi\right) \\
& R_{M}=R_{e}\left(1-2 e+3 e \sin ^{2} \varphi\right) \\
& g=9.80665+0.051799 \sin ^{2} \varphi-3.086 \times 10^{-6} h
\end{aligned}
$$

In the Eq.(3), $R_{e}=6378137.0 \mathrm{~m}$ is the semi-major axis of the earth, $e=1 / 298.257$ is the eccentricity of earth and $h$ is the altitude.

The differential equation of position is shown in Eq.(4).

$$
\dot{\varphi}=\frac{V_{N}}{R_{M}+h} \quad \dot{\lambda}=\frac{V_{E}}{\left(R_{N}+h\right) \cos \varphi} \quad \dot{h}=V_{U}
$$

In the Eq.(4), $\lambda$ is the longitude. 


\section{Tightly-Coupled Error State Covariance Shaping Adaptive Kalman Filter}

A tightly-coupled error-state extended Kalman filter with a centralized architecture has been proposed by Groves[10], but its computation is so complex that it's not conducive for the application in the project or promotion. In order to solve this problem, a scheme of distributed filters with 'speed channel' and 'position channel' is been proposed in this paper, which can depress the error divergence of INS by the speed feedback correction. The architecture of the tightly coupled GNSS/MIMU integration system can be seen in Figure.1.

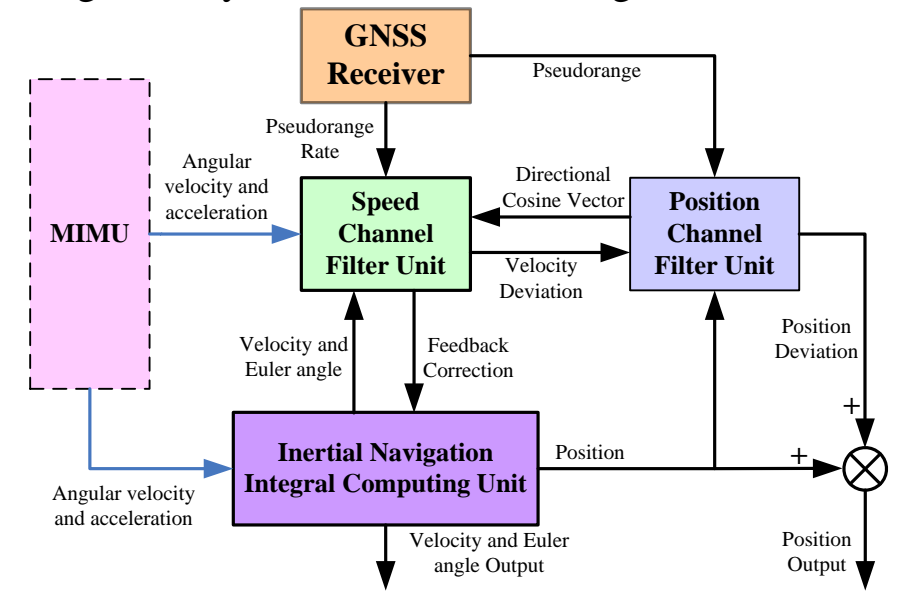

Fig.1. Tightly Coupled GNSS/MIMU Integration Architecture

\section{System State Model of Adaptive Kalman Filter}

Using the position and speed differences between GNSS observables and INS predicted observables, the Kalman filter is used to estimate the INS solution errors with the state vector as shown in Eq.(5).

$$
\begin{aligned}
& \boldsymbol{x}^{p}=\left[\delta \varphi, \delta \lambda, \delta h, \delta t_{u}, \eta_{1}, \eta_{2}, \ldots, \eta_{n}\right]^{T} \\
& \boldsymbol{x}^{v}=\left[\delta V_{N}, \delta V_{U}, \delta V_{E}, \delta \dot{t}_{u}, \psi_{N}, \psi_{U}, \psi_{E}, \varepsilon_{x}, \varepsilon_{y}, \varepsilon_{z}, \sigma_{x}, \sigma_{y}, \sigma_{z}\right]^{T}
\end{aligned}
$$

In the Eq.(5), the superscript $p$ and $v$ respectively represents the position channel filter unit and the speed channel filter unit; $\delta \varphi, \delta \lambda, \delta h$ is the estimated latitude, longitude and height error respectively; $\delta t_{u}$ is the estimated clock bias of the receiver; $\eta_{i}(i=1,2, \cdots, n)$ is the estimated phase bias of each satellite in view; $\delta V_{N}, \delta V_{U}, \delta V_{E}$ is respectively the estimated speed error; $\delta \dot{t}_{u}$ is the estimated drift rate of the receiver clock bias; $\varepsilon_{x}, \varepsilon_{y}, \varepsilon_{z}$ is respectively the estimated gyro bias; $\sigma_{x}, \sigma_{y}, \sigma_{z}$ is respectively the estimated accelerometer bias; and $\psi_{N}, \psi_{U}, \psi_{E}$ is respectively the estimated attitude error defined in Eq.(6).

$$
\boldsymbol{I}+\boldsymbol{\psi}^{\times}=\boldsymbol{C}_{b}^{n^{*}} \boldsymbol{C}_{n}^{b} \quad \text { and } \quad \boldsymbol{\psi}^{\times}=\left[\begin{array}{ccc}
0 & -\psi_{E} & \psi_{U} \\
\psi_{E} & 0 & -\psi_{N} \\
-\psi_{U} & \psi_{N} & 0
\end{array}\right]
$$

In the Eq.(6), $\boldsymbol{C}_{n}^{b}$ is the direction cosine matrix (DCM) without deviation, $\boldsymbol{C}_{b}^{n^{*}}$ is the DCM with deviation, and $\boldsymbol{I}$ is the identity matrix.

The discrete state equation is shown in Eq.(7).

$$
\begin{aligned}
& \boldsymbol{x}_{k+1}^{p}=\boldsymbol{x}_{k}^{p}+\boldsymbol{B}_{k}^{p} \boldsymbol{u}_{k}^{p}+\boldsymbol{w}_{k}^{p} \\
& \boldsymbol{x}_{k+1}^{v}=\boldsymbol{A}_{k}^{v} \boldsymbol{x}_{k}^{v}+\boldsymbol{w}_{k}^{v} \\
& \boldsymbol{u}_{k}^{p}=\left[\delta V_{N}, \delta V_{U}, \delta V_{E}, 0_{1 \times n}\right]^{T}
\end{aligned}
$$

In the Eq.(7), $\boldsymbol{x}_{k}^{p}, \boldsymbol{x}_{k+1}^{p}$ and $\boldsymbol{x}_{k}^{v}, \boldsymbol{x}_{k+1}^{v}$ is respectively the state vector in time $t_{k}, t_{k+1} ; \boldsymbol{u}_{k}^{p}$ is the input vector given by the speed channel filter unit; $\boldsymbol{B}_{k}^{p}$ is the driving matrix; $\boldsymbol{w}_{k}^{p}, \boldsymbol{w}_{k}^{v}$ is the 
process noise with zero mean respectively; $\boldsymbol{A}_{k}^{v}$ is the transition matrix, which can be acquired through the error propagation model of INS. Eq.(8) presents the details of $\boldsymbol{A}_{k}^{v}$.

$$
\boldsymbol{A}_{k}^{v}=\left[\begin{array}{ccccc}
\boldsymbol{I}_{3 \times 3} & \delta \boldsymbol{V}^{n} \times & 0_{3 \times 3} & 0_{3 \times 3} & \boldsymbol{C}_{b}^{n} \delta \boldsymbol{t} \\
0 & 1 & 0 & 0 & 0 \\
0_{3 \times 3} & 0_{3 \times 3} & \boldsymbol{I}_{3 \times 3} & \boldsymbol{C}_{b}^{n} \delta \boldsymbol{t} & 0_{3 \times 3} \\
0_{3 \times 3} & 0_{3 \times 3} & 0_{3 \times 3} & \boldsymbol{I}_{3 \times 3} & 0_{3 \times 3} \\
0_{3 \times 3} & 0_{3 \times 3} & 0_{3 \times 3} & 0_{3 \times 3} & \boldsymbol{I}_{3 \times 3}
\end{array}\right] \quad \delta \boldsymbol{V}^{n} \times=\left[\begin{array}{ccc}
0 & -\Delta V_{E} & \Delta V_{U} \\
\Delta V_{E} & 0 & -\Delta V_{N} \\
-\Delta V_{U} & \Delta V_{N} & 0
\end{array}\right]
$$

$\boldsymbol{B}_{k}^{p}$ is a sparse matrix, which can be acquired through the error propagation model of INS. And its nonzero elements are shown in Eq.(9).

$$
\boldsymbol{B}_{k}^{p}(1,4)=\frac{\delta t}{R_{M}+h} \quad \boldsymbol{B}_{k}^{p}(2,6)=\frac{\delta t}{\left(R_{N}+h\right) \cos \varphi} \quad \boldsymbol{B}_{k}^{p}(3,5)=\delta t
$$

In the Eq.(9), $\delta t$ is the time step for the state updating.

\section{System Measurement Model of Adaptive Kalman Filter}

The linearized measurement model is:

$$
\begin{aligned}
\mathbf{z}_{k}^{p} & =\boldsymbol{H}_{k}^{p} \boldsymbol{x}_{k}^{p}+\boldsymbol{v}_{p} \\
\mathbf{z}_{k}^{v} & =\boldsymbol{H}_{k}^{v} \boldsymbol{x}_{k}^{v}+\boldsymbol{v}_{v}
\end{aligned}
$$

In the Eq.(10), $\mathbf{z}_{k}^{p}, \mathbf{z}_{k}^{v}$ is respectively the position measurement vector, $\boldsymbol{H}_{k}^{p}, \boldsymbol{H}_{k}^{v}$ is respectively the observation matrix of position channel, and $\boldsymbol{v}_{p}, \boldsymbol{v}_{v}$ is respectively a measurement vector of the random noise with zero mean and $\boldsymbol{R}_{p}, \boldsymbol{R}_{v}$ covariance.

In the tightly-coupled architecture, the available observations are measurements of pseudoranges and pseudo-range rates. Measurement model can be modeled as follow:

$$
\begin{aligned}
& \mathbf{z}_{k}^{p}=\left[\begin{array}{c}
\rho_{1}^{m}-\rho_{1}^{c} \\
\rho_{2}^{m}-\rho_{2}^{c} \\
\vdots \\
\rho_{n}^{m}-\rho_{n}^{c}
\end{array}\right]=\left[\begin{array}{c}
\cos u_{1} \\
\cos u_{2} \\
\vdots \\
\cos u_{n}
\end{array}\right]\left[\begin{array}{ccc}
-R_{N}^{h} \sin \varphi \cos \lambda & -R_{N}^{h} \cos \varphi \sin \lambda & \cos \varphi \cos \lambda \\
-R_{N}^{h} \sin \varphi \sin \lambda & R_{N}^{h} \cos \varphi \cos \lambda & \cos \varphi \sin \lambda \\
\left(R_{N} a^{2} / b^{2}+h\right) \cos \varphi & 0 & \sin \varphi
\end{array}\right]\left[\begin{array}{c}
\delta \varphi \\
\delta \lambda \\
\delta h
\end{array}\right]-\left[\begin{array}{c}
\delta t_{u} \\
\delta t_{u} \\
\vdots \\
\delta t_{u}
\end{array}\right]+\left[\begin{array}{c}
\eta_{1} \\
\eta_{2} \\
\vdots \\
\eta_{n}
\end{array}\right] \\
& \mathbf{z}_{k}^{v}=\left[\begin{array}{c}
\dot{\rho}_{1}^{m}-\dot{\rho}_{1}^{c} \\
\dot{\rho}_{2}^{m}-\dot{\rho}_{2}^{c} \\
\vdots \\
\dot{\rho}_{n}^{m}-\dot{\rho}_{n}^{c}
\end{array}\right]=\left[\begin{array}{c}
\cos u_{1} \\
\cos u_{2} \\
\vdots \\
\cos u_{n}
\end{array}\right]\left[\begin{array}{ccc}
-\sin \varphi \cos \lambda & \cos \varphi \cos \lambda & -\sin \lambda \\
-\sin \varphi \sin \lambda & \cos \varphi \sin \lambda & \cos \lambda \\
\cos \varphi & \sin \varphi & 0
\end{array}\right]\left[\begin{array}{c}
\delta V_{N} \\
\delta V_{U} \\
\delta V_{E}
\end{array}\right]-\left[\begin{array}{c}
\delta \dot{t}_{u} \\
\delta \dot{t}_{u} \\
\vdots \\
\delta \dot{t}_{u}
\end{array}\right]
\end{aligned}
$$

In the Eq.(11), $\rho_{i}^{m}$ is the pseudo-range measurement of GNSS receiver, $\dot{\rho}_{i}^{m}$ is the pseudo-range rate measurement of the GNSS receiver, $\rho_{i}^{c}$ is the pseudo-range and $\dot{\rho}_{i}^{c}$ is the pseudo-range rate calculated by Eq. (12), and $\boldsymbol{c o s u}_{i}$ is a cosine vector directed from the GNSS receiver to the GNSS satellite with the following form:

$$
\begin{gathered}
\operatorname{cosu}_{i}=\left[\frac{x_{0}-x_{i}}{\rho_{i}}, \frac{y_{0}-y_{i}}{\rho_{i}}, \frac{z_{0}-z_{i}}{\rho_{i}}\right]^{T} \\
\rho_{i}^{c}=\sqrt{\left(x_{0}-x_{i}\right)^{2}+\left(y_{0}-y_{i}\right)^{2}+\left(z_{0}-z_{i}\right)^{2}} \quad \dot{\rho}_{i}^{c}=\operatorname{cosu}_{i} \cdot\left(\boldsymbol{v}_{i}^{s}-\boldsymbol{v}^{n}\right)
\end{gathered}
$$

In the Eq.(12), $\rho_{i}$ is the GNSS pseudo-range measurement vector with receiver clock error correcton; $x_{0}, y_{0}, z_{0}$ is respectively the position of INS in the earth-centered earth-fixed(ECEF) coordinate; $x_{i}, y_{i}, z_{i}$ is respectively the $i$ th GNSS satellite position in ECEF; $\boldsymbol{v}^{n}$ is the INS velocity vector in ECEF and $\boldsymbol{v}_{i}^{s}$ is the $i$ th GNSS satellite velocity vector in ECEF. 


\section{Covariance Shaping Adaptive Kalman Filter}

The ratios of process noise covariance and measurement noise covariance determine the Kalman filter bandwidth(namely the convergence rate), which is directly related to the performance of the filter. The residual of measurement reflects the performance of Kalman filter and when it is a Gaussian white sequence the filter is optimal. In other words, the residual of measurement contains the necessary information of the filter error propagation which makes it a very good candidate for the filter self-tuning or adaptation. In order to optimize the convergence rate and estimation accuracy, it's necessary to estimate the optimal process noise covariance in real time. The process noise covariance can be decoupled into two parts. One, the same as the traditional filter, has a fixed initial error covariance and updates periodically. The other forms the cost function with the residuals of measurements, and then estimates the weighting gain of the error covariance in real time to minimize the cost function. It varies along with the error of the measurements' residuals. Here, the Frobenius norm is used as the form of cost function. The state-space model of GNSS/MIMU tightly-coupled integrated navigation system is given by a discrete-time linear equation as:

$$
\begin{aligned}
\dot{\boldsymbol{x}}_{k} & =\boldsymbol{A}_{k-1, k} \boldsymbol{x}_{k-1}+\boldsymbol{w}_{k-1} \\
\boldsymbol{z}_{k} & =\boldsymbol{H}_{k} \boldsymbol{x}_{k}+\boldsymbol{v}_{k}
\end{aligned}
$$

The residual of measurement can be derived from the measurement itself and the estimated measurement from the filter.

$$
\boldsymbol{e}_{k}=\boldsymbol{H}_{k}\left(\boldsymbol{x}_{k}-\hat{\boldsymbol{x}}_{k}\right)+\boldsymbol{v}_{k}=\boldsymbol{H}_{k} \delta \boldsymbol{x}_{k}+\boldsymbol{v}_{k}
$$

In the Eq.(14), $\boldsymbol{e}_{k}$ is the residual of measurement and $\delta \boldsymbol{x}_{k}$ is the estimation error. And the covariance of the residual of measurement is described as

$$
\boldsymbol{S}_{k-1, k}=E\left[\boldsymbol{e}_{k} \cdot \boldsymbol{e}_{k}^{T}\right]=\boldsymbol{H}_{k} \boldsymbol{P}_{k-1, k} \boldsymbol{H}_{k}^{T}+\boldsymbol{R}_{k}
$$

In the Eq.(15), $\boldsymbol{R}_{k}$ is the covariance of measurement noise. And the error covariance matrix can be written as

$$
\boldsymbol{P}_{k-1, k}=\boldsymbol{P}_{k-1, k}^{0}+\alpha \boldsymbol{P}_{k-1, k}^{1}
$$

In the Eq.(16), $\alpha$ is the adaptive gain matrix to minimize the cost function. Substituting Eq.(16) into Eq.(15), the perturbed covariance of residual of measurement is achieved as follows.

$$
\begin{aligned}
\boldsymbol{S}_{k-1, k} & =\boldsymbol{H}_{k}\left(\boldsymbol{P}_{k-1, k}^{0}+\alpha \boldsymbol{P}_{k-1, k}^{1}\right) \boldsymbol{H}_{k}^{T}+\boldsymbol{R}_{k} \\
& =\boldsymbol{H}_{k} \boldsymbol{P}_{k-1, k}^{0} \boldsymbol{H}_{k}^{T}+\boldsymbol{H}_{k} \alpha \boldsymbol{P}_{k-1, k}^{1} \boldsymbol{H}_{k}^{T}+\boldsymbol{R}_{k}=\boldsymbol{S}_{k-1, k}^{0}+\boldsymbol{S}_{k-1, k}^{1} \\
\boldsymbol{S}_{k-1, k}^{0} & =\boldsymbol{H}_{k} \boldsymbol{P}_{k-1, k}^{0} \boldsymbol{H}_{k}^{T}+\boldsymbol{R}_{k} \quad \boldsymbol{S}_{k-1, k}^{1}=\boldsymbol{H}_{k} \alpha \boldsymbol{P}_{k-1, k}^{1} \boldsymbol{H}_{k}^{T}
\end{aligned}
$$

The above equation means that the adaptive gain can reset the error covariance with respect to the measurement variation. The average covariance of residuals of measurements with $N$ samples is given as

$$
\overline{\boldsymbol{S}}=\frac{1}{N} \sum_{i=1}^{N}\left[\delta \mathbf{z}(k+1-N+i) \delta \mathbf{z}(k+1-N+i)^{T}\right]
$$

Eq.(17) is the covariance of residuals of measurements estimated from Kalman filter and Eq.(18) is the covariance of residuals of measurements in real time. Thus we can derive the cost function which is used to minimize the difference between Eq.(17) and Eq.(18), and estimate the optimal $\alpha$ in real time. The cost function is defined by the Frobenius norm, and $\alpha$ is estimated to minimize this norm value.

Denote $\|\cdot\|$ as the Frobenius norm and $\|S\|^{2}=\operatorname{tr}\left(S S^{T}\right)$. Then the cost function is defined as

$$
\min _{\alpha>0}\left\{J(\alpha)=\left\|\overline{\boldsymbol{S}}-\left(\boldsymbol{S}_{k-1, k}^{0}+\boldsymbol{S}_{k-1, k}^{1}\right)\right\|^{2}\right\}
$$

In the Eq.(19), $J(\alpha)$ is evaluated for $\alpha$ to minimize the cost function. According to the denotation of the Frobenius norm, here we have 


$$
\begin{aligned}
& J(\alpha)=\operatorname{tr}\left[\left(\overline{\boldsymbol{S}}-\boldsymbol{S}_{k-1, k}^{0}\right)\left(\overline{\boldsymbol{S}}-\boldsymbol{S}_{k-1, k}^{0}\right)^{T}-2 \beta\left(\overline{\boldsymbol{S}}-\boldsymbol{S}_{k-1, k}^{0}\right)^{T}+\beta \beta^{T}\right] \\
& \beta=\boldsymbol{H} \alpha \boldsymbol{P}_{k-1, k}^{1} \boldsymbol{H}^{T}=\left[\begin{array}{cccc}
\alpha_{11} P_{11} & \beta_{12} & \cdots & \beta_{1 m} \\
\beta_{21} & \alpha_{22} P_{11} & \cdots & \beta_{2 m} \\
\vdots & \vdots & \ddots & \vdots \\
\beta_{m 1} & \beta_{m 2} & \cdots & \alpha_{m m} P_{m m}
\end{array}\right]
\end{aligned}
$$

$\beta$ is an antisymmetric matrix. Differentiating Eq.(20) with $\alpha$,

$$
\frac{\partial J(\alpha)}{\partial \alpha}=-2\left[\frac{\partial \beta}{\partial \alpha}\left(\overline{\boldsymbol{S}}-\boldsymbol{S}_{k-1, k}^{0}\right)^{T}-\frac{\partial \beta}{\partial \alpha} \beta^{T}\right]
$$

In order to minimize $J(\alpha)$,

$$
\frac{\partial J(\alpha)}{\partial \alpha}=0 \Rightarrow \alpha=\operatorname{diag}\left[\left(\boldsymbol{H P}_{k-1, k}^{1} \boldsymbol{H}^{T}\right)^{-1}\left(\overline{\boldsymbol{S}}-\boldsymbol{S}_{k-1, k}^{0}\right)\right]
$$

So, we can optimize the Kalman filter by turning $\alpha$ calculate by Eq.(22).

\section{Test Results and Discussions}

The hardware-in-the-loop simulation results are presented to prove the effectiveness of the

\begin{tabular}{|c|c|}
\hline Items & Parameters \\
\hline $\begin{array}{l}\text { GNSS } \\
\text { Receiver }\end{array}$ & $\begin{array}{l}\text { Pseudo-range precision: } 6 \mathrm{~m}(1 \sigma) \text {; Pseudo-range rate precision: } 0.15 \mathrm{~m} / \mathrm{s}(1 \sigma) \text {; Data } \\
\text { frequency: } 10 \mathrm{~Hz}\end{array}$ \\
\hline MIMU & $\begin{array}{l}\text { Gyro In-Run Bias Stability: } 10^{\circ} / \mathrm{h}(1 \sigma) \text {; Gyro Bias Repeatability(typ): } 0.5^{\circ} / \mathrm{s} \text {; Gyro } \\
\text { Sensitivity Tempco(typ): } 50 \mathrm{ppm} /{ }^{\circ} \mathrm{C} \text {; Accelerometer In-Run Bias Stability: } \\
0.5 \mathrm{mg}(1 \sigma) \text {; Accelerometer Bias Repeatability(typ): } 2.0 \mathrm{mg} \text {; Accelerometer } \\
\text { Sensitivity Tempco: } 20 \mathrm{ppm} /{ }^{\circ} \mathrm{C}\end{array}$ \\
\hline
\end{tabular}
proposed methods in this paper. The parameters and technical indexes of the GNSS receiver and MIMU are shown in Table 1.

Table 1. The parameters of GNSS receiver and MIMU

The hardware composition can be seen in Figure 2.
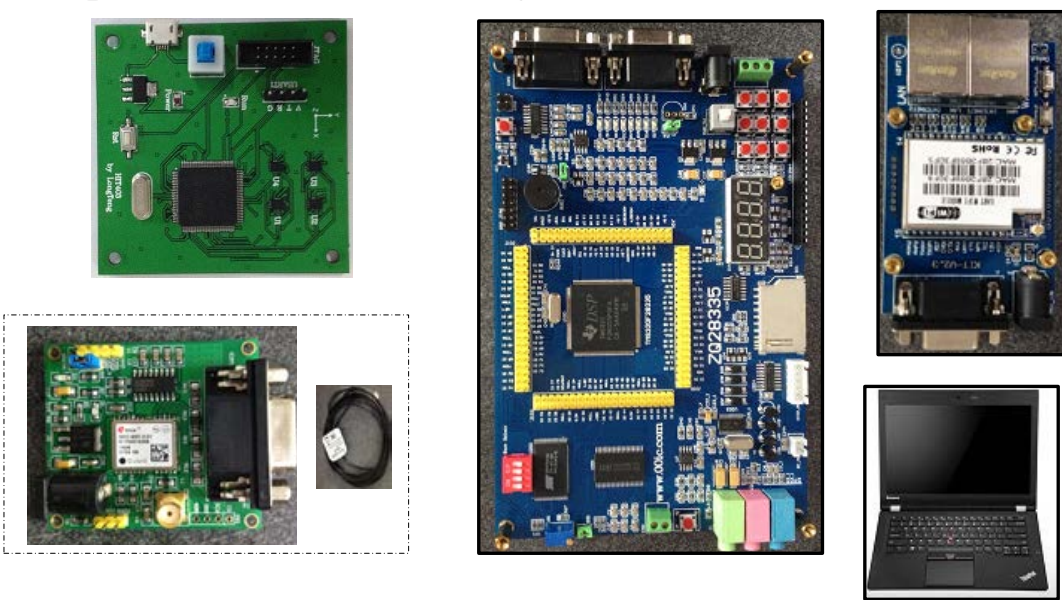

Figure 2. The hardware composition of the simulation system. (1) Self-designed development board for multi-sensors redundant system with four BOSCH BMI160 MIMUs and one STM32F407VET6 168MHz ARM. (2) Self-designed development board for one Ublox NEO-M8T GNSS receiver. (3) Development kit for TMS320F28335 Digital Signal Processor(DSP). (4) HLK RM-04 UART-WIFI Module. (5) Data fusion computer for integrated navigation system. 

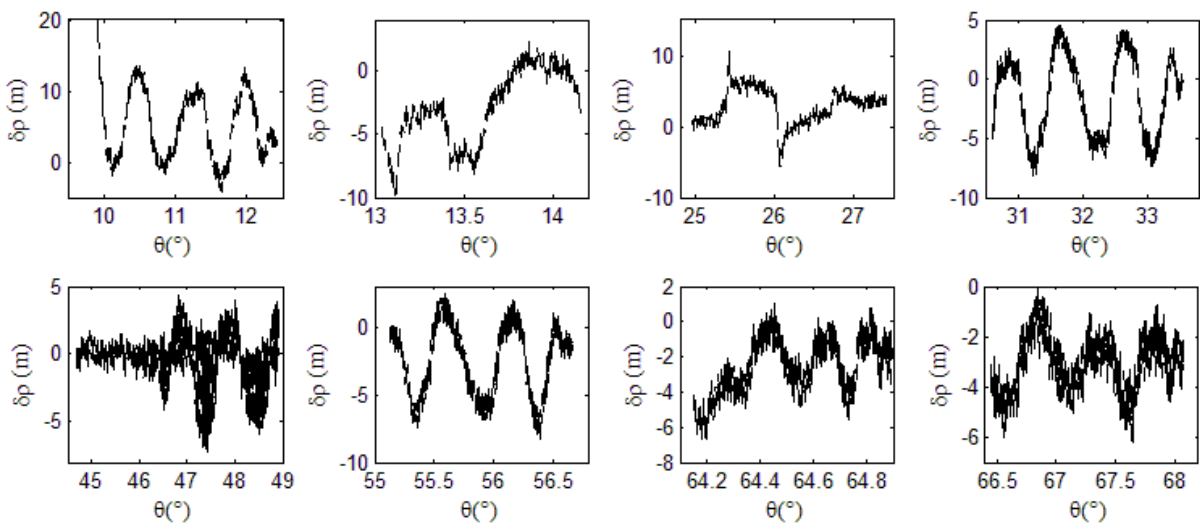

Figure 3. The curve of satellite elevation angle and pseudo-range deviation

The relationships between the satellite elevation angle and the pseudo-range deviation of GNSS receiver measurements can be seen in Figure 3. Those figures show that the higher the satellite elevation angle is, the higher the accuracy of pseudo-range can be. The pseudo-range measurement of GNSS receiver has rapid changes of the measurement error and its distibution is not the gauss distribution, which cause the standard Kalman filter can not achieve the optimal estimation.

In order to achieve the optimal estimation, the covariance shaping in real time is needed. The simulation comparisons between the variance shaping adaptive Kalman filter and standard Kalman filter are shown in Figure 4.
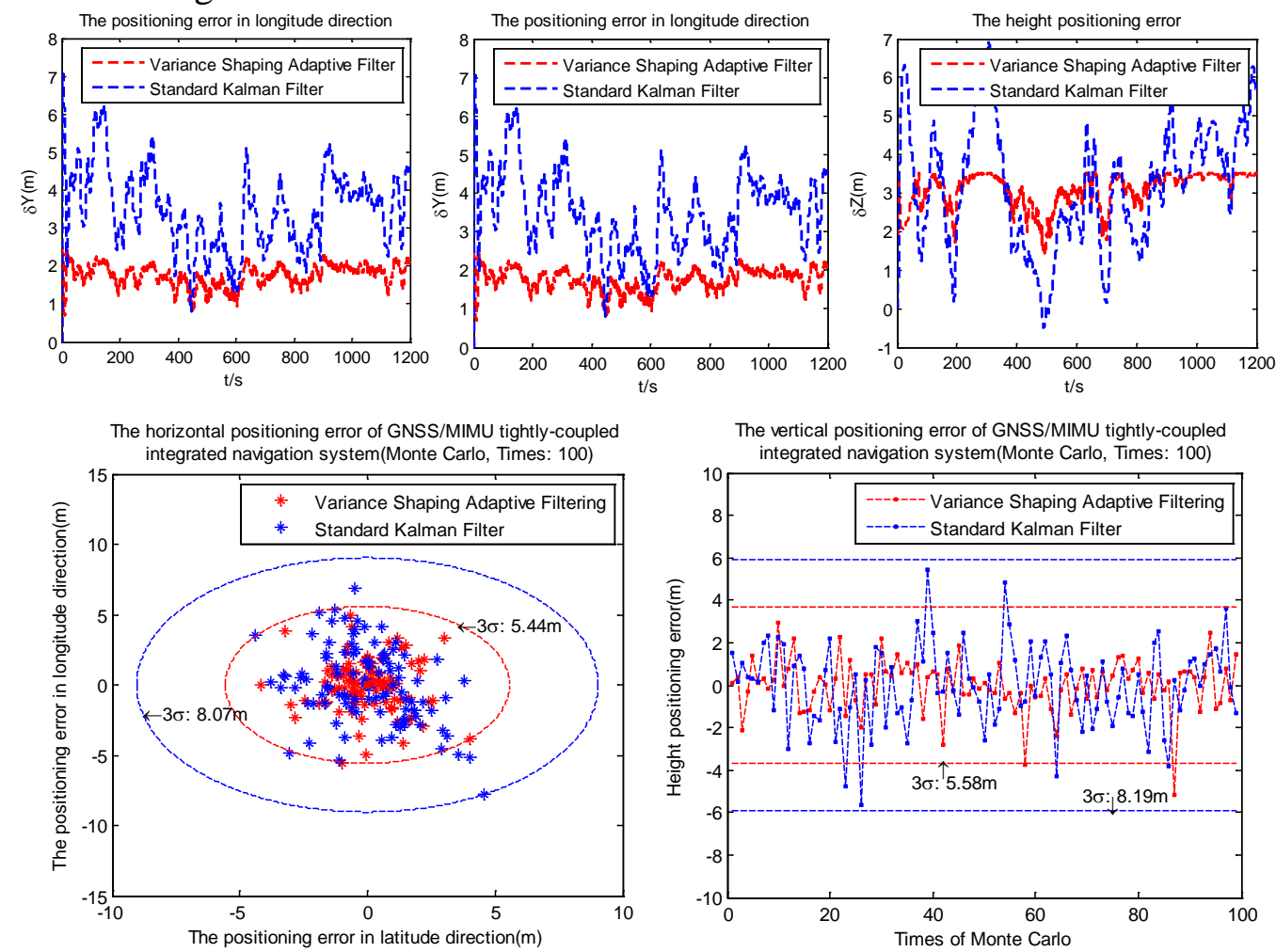

Figure 4. The simulation results of the variance shaping adaptive Kalman filter and standard Kalman filter. (1) The latitude positioning error. (2) The longitude positioning error. (3) The height positioning error. (4) The horizontal positioning statistical accuracy with 100 Monte-Carlo targeting.

(5) The vertical positioning statistical accuracy with 100 Monte-Carlo targeting.

As shown in Figure 4, the positioning accuracy of GNSS/MIMU tightly-coupled integrated navigation system with the variance shaping adaptive Kalman filter is increased by approximately $32.6 \%$ compared with the standard Kalman filter. At the same time, the stability of the variance shaping adaptive filter has much better performance, when the measurement noises of the pseudo-range change violently(as shown in Figure.3). The output data of the variance shaping adaptive filter is smoother than the standard Kalman filter, but one or two orders digital filter is 
needed to add for the much smoother data output.

\section{Conclusion}

This article presents a novel estimator to optimize the computational complexity and the positioning accuracy. A covariance shaping adaptive filtering method and dual channel parallel filter architecture with 'position channel' and 'speed channel' are proposed for the MIMU/GNSS tightly-coupled inertial navigation system, which can improve the accuracy about $32.6 \%$ proved by the hardware-in-the-loop simulation tests. In the covariance shaping adaptive filter, the process noise covariance is divided into the fixed part and variable part, and the filter is optimized by minimizing the cost function, which is in the form of the Frobenius norm. The adaptive gain of the error covariance is estimated in accordance with the measurement change, and resets the error covariance into new valid values in harmony with the measurement. The simulation results demonstrate the estimation accuracy under the inadequate measurement noise and high dynamics.

\section{Acknowledgement}

In this paper, the research was sponsored by the National High Technology Research and Development Program of China (Project No. 2015AA7026083).

\section{References}

[1] Rabbou M A, El-Rabbany A. Tightly coupled integration of GPS precise point positioning and MEMS-based inertial systems[J]. GPS Solutions, 2015, 19(4): 601-609.

[2] Chiang K W, Lin C A, Peng K Y. The performance analysis of an AKF based tightly-coupled INS/GNSS sensor fusion scheme with non-holonomic constraints for land vehicular applications[C]. Applied Mechanics and Materials, 2013, 284: 1956-1960.

[3] Wendel J, Trommer G F. Tightly coupled GPS/INS integration for missile applications[J]. Aerospace Science and Technology, 2004, 8(7): 627-634.

[4] Benzerrouk H, Nebylov A V. Integrated navigation system INS/GNSS based on joint application of linear and nonlinear filtering[J]. IFAC Proceedings Volumes, 2012, 45(1): 208-213.

[5] George M, Sukkarieh S. Tightly coupled INS/GPS with bias estimation for UAV applications[C]. Proceedings of Australiasian Conference on Robotics and Automation (ACRA), 2005.

[6] Rieke M, Foerster T, Geipel J, et al. High-precision positioning and real-time data processing of UAV systems[J]. International Archives of the Photogrammetry, Remote Sensing and Spatial Information Sciences, 2011, 38: 1.

[7] Mathieu J L, Koch S, Callaway D S. State estimation and control of electric loads to manage real-time energy imbalance[J]. IEEE Transactions on Power Systems, 2013, 28(1): 430-440.

[8] Goyal V, Deolia V K, Sharma T N. Neural network based sliding mode control for uncertain discrete-time nonlinear systems with time-varying delay[J]. International Journal of Computational Intelligence Research, 2016, 12(2): 125-138.

[9] Shi E. An improved real-time adaptive Kalman filter for low-cost integrated GPS/INS navigation[C]. Measurement, Information and Control (MIC), 2012 International Conference on. IEEE, 2012, 2: 1093-1098.

[10] Groves P D. Principles of GNSS, inertial, and multi-sensor integrated navigation systems[M]. Artech house, 2013. 